Delft University of Technology

\title{
Estimation of Facial Contact Pressure Based on Finite Element Analysis
}

Lee, Wonsup; Kim, Jin-Gyun; Molenbroek, Johan M.F.; Goossens, Richard H.M.; You, Heecheon

$\mathrm{DOI}$

10.1007/978-3-030-20216-3_61

Publication date

2020

Document Version

Final published version

Published in

Advances in Additive Manufacturing, Modeling Systems and 3D Prototyping - Proceedings of the AHFE 2019 International Conference on Additive Manufacturing, Modeling Systems and 3D Prototyping

\section{Citation (APA)}

Lee, W., Kim, J-G., Molenbroek, J. M. F., Goossens, R. H. M., \& You, H. (2020). Estimation of Facial

Contact Pressure Based on Finite Element Analysis. In M. Di Nicolantonio, E. Rossi, \& T. Alexander (Eds.), Advances in Additive Manufacturing, Modeling Systems and 3D Prototyping - Proceedings of the AHFE 2019 International Conference on Additive Manufacturing, Modeling Systems and 3D Prototyping: Proceedings of the AHFE 2019 International Conference on Additive Manufacturing, Modeling Systems and 3D Prototyping (1 ed., Vol. 975, pp. 657-667). (Advances in Intelligent Systems and Computing; Vol. 975). Springer Science+Business Media. https://doi.org/10.1007/978-3-030-20216-3_61

Important note

To cite this publication, please use the final published version (if applicable).

Please check the document version above.

\section{Copyright}

Other than for strictly personal use, it is not permitted to download, forward or distribute the text or part of it, without the consent of the author(s) and/or copyright holder(s), unless the work is under an open content license such as Creative Commons.

\section{Takedown policy}

Please contact us and provide details if you believe this document breaches copyrights.

We will remove access to the work immediately and investigate your claim. 
Green Open Access added to TU Delft Institutional Repository 'You share, we take care!' - Taverne project

\section{https://www.openaccess.nl/en/you-share-we-take-care}

Otherwise as indicated in the copyright section: the publisher is the copyright holder of this work and the author uses the Dutch legislation to make this work public. 


\title{
Estimation of Facial Contact Pressure Based on Finite Element Analysis
}

\author{
Wonsup Lee ${ }^{1(\bowtie)}$, Jin-Gyun Kim² ${ }^{2}$, Johan M. F. Molenbroek ${ }^{3}$, \\ Richard H. M. Goossens ${ }^{3}$, and Heecheon $\mathrm{You}^{4}$ \\ ${ }^{1}$ School of Global Entrepreneurship and Information Communication \\ Technology, Handong Global University, Pohang 37554, South Korea \\ W. Lee@Handong. edu \\ 2 Department of Mechanical Engineering, Hyung Hee University, \\ Yongin 17104, South Korea \\ Jingyun.Kim@KHU. ac.kr \\ ${ }^{3}$ Faculty of Industrial Design Engineering, Delft University of Technology, \\ 2628 CE Delft, The Netherlands \\ \{J.F.M.Molenbroek, R.H.M.Goossens\}@TUDelft.nl \\ ${ }^{4}$ Department of Industrial and Management Engineering, Pohang University of \\ Science and Technology, Pohang 37673, South Korea \\ hcyou@Postech.edu
}

\begin{abstract}
A 3D head and face scan data can be usefully applied in the ergonomic design of a wearable product such as an oxygen mask or a virtual reality (VR) headset to support the safety as well as comfort for a certain amount of a target population. This study is aimed to develop analysis and design methods of an ergonomic facial wearable products (e.g., pilot oxygen mask, VR headsets) based on numerous 3D face scan data (366 faces and 2,299 heads) through the template registration and the finite element (FE) analysis methods to predict the contact pressure between the face and a product. The application of the FE analysis on numerous $3 \mathrm{D}$ images to find an optimal form of a product is a novel approach in product design. The proposed methods on contact pressure analysis could be usefully applied for wearable products, which fit body parts.
\end{abstract}

Keywords: Contact pressure estimation $\cdot$ Face $\cdot$ Finite element analysis $\cdot$ Ergonomic product design

\section{Introduction}

Technologies of 3D human body scanning and analysis have been emerged during the last decade and usefully applied in the ergonomic product design as well as orthopedics, plastic surgery, healthcare, fashion, film making, game, and so forth. A 3D scan image of a human can provide various useful information for product design including anthropometric body dimensions (e.g., length, width, circumference, and any other point-to-point or surface lengths) and body shape information (e.g., arc, cross-sectional curvature, surface, area, and volume). A product designed based on those 3D anthropometric and shape information could be resulted in gaining a higher satisfaction in terms of fit, comfort, safety, and usability. Particularly, a facial wearable product 
such as masks (e.g., medical mask, dust-proof mask, gas mask, protective mask, sports mask) and goggles (e.g., protective goggle, sports goggle, virtual reality headset) needs to have an ergonomically designed form that can fit well to the faces of a target user population.

An optimal shape of a facial product design can be found by analyzing facial anthropometric and shape characteristics as well as the contact pressure between the product and the face. Lee et al. [1,2] proposed a method of computer-based analysis of the fit between the faces $(n=336)$ and a pilot oxygen mask for Korean Air Force pilots to find the best form of the oxygen mask, which fits the high amount of Korean pilots. In their follow-up study, the finite element (FE) analysis technique was applied to estimate the contact pressure between the faces and the pilot oxygen mask in order to derive an optimal shape of the oxygen mask that shows a better fit to Korean pilots compared to the previous design [3]. The FE-based facial contact pressure analysis has been introduced by previous studies [4-6]. While the previous studies used only a few head models (say, less than five) for the pressure estimation of a mask product, this study had been tried to use hundreds and thousands of heads to calculate the contact pressure characteristics of a population that includes people showing different sizes and shapes of the head.

This study is aimed to introduce a method and a case study of an ergonomic wearable product design based on numerous 3D body scan database and FE analysis technology. The faces of Korean Air Force pilots $(n=336)$ and the heads of Americans $(n=2,299)$ were used to analyze the contact pressure of the pilot oxygen mask and virtual reality (VR) headsets.

\section{Methods}

\subsection{D Face Scans}

3D face scan images of Korean Air Force pilots (male: 278, female: 58; age: 20s to 40s) collected by Lee et al. [7] and 3D head images of Americans (male: 1,086, female: 1,213; age: 10s to 60s; Caucasian: 1,818, African American: 258, Asian: 173, Hispanic: 50) derived from Civilian American and European Surface Anthropometry Resource (CAESAR) database [8] were used in this study.

The post-processing process including editing, landmarking, and alignment of 3D models was applied on 3D face images. 14 and 26 anthropometric landmarks were respectively identified to the 3D pilots' face and the American heads. All the faces and heads were aligned in the 3D coordinates with the origin point at the sellion landmark and two vectors (one vertical vector parallel to the $\mathrm{Y}$-axis and passing through sellion and supramentale and one horizontal vector parallel to the $\mathrm{X}$-axis and passing through left and right tragion) [2].

\subsection{D Face Scans}

A template face and head models sophisticatedly edited to have well-distributed meshes with an appropriate number of vertex were prepared with consideration of its 

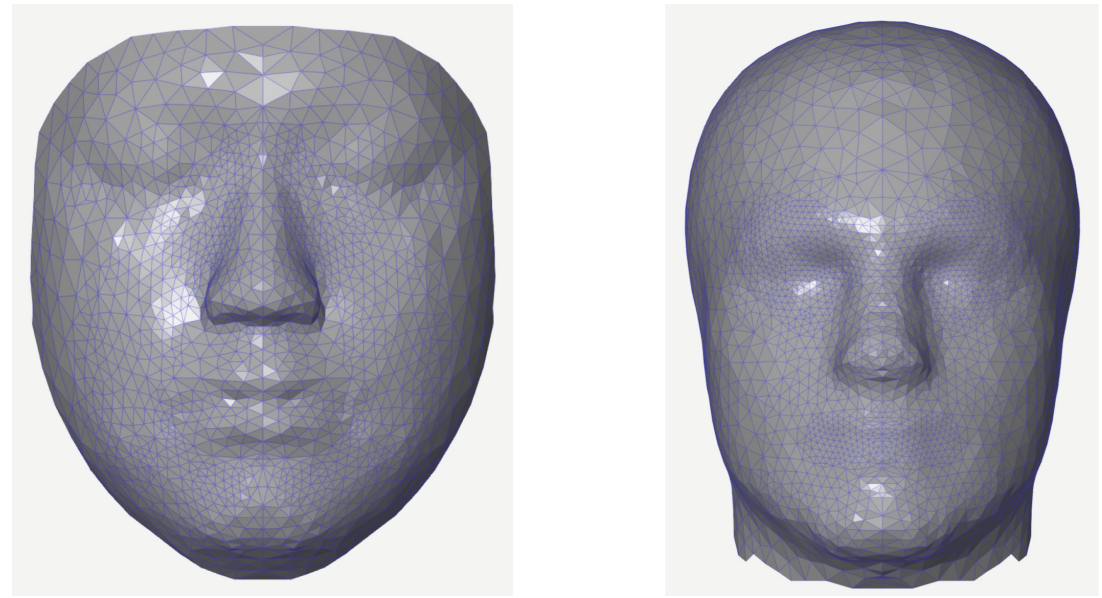

Fig. 1. Face (left) and head (right) template models.

applicability in the FE analysis, then the face and head templates were registered to each of the 3D faces and heads, respectively. Template registration, one of a well-used method in the computer graphics, was used in this study to makes different 3D scans having the same vertex points and mesh structure through the following procedure. First, symmetrized template models (face model: 1,340 vertex points and 2,624 meshes; head model: 2,787 vertex points and 5,532 meshes) were sophisticatedly generated by hand (see Fig. 1) using the RapidForm 2006 (INUS Technology, Inc., Korea) image processing software. Second, template model registration was conducted by applying a hybrid approach proposed by Lee et al. [3, 9], which used the bounded biharmonic weights (BBW) mesh deformational algorithm [10] and the non-rigid iterative closest point (ICP) registration technique [11] in this study. The BBW were used to roughly change the size of the template model by matching its landmark locations to a target image's landmark locations. In the BBW method, weight values are assigned to each vertex point of the template by considering distances between a vertex point and a landmark location. When the landmarks of the template model are relocated to positions of the corresponding landmarks of a target image through the BBW deformation, all vertex points around each landmark of the template model are also relocated to be designated locations by referring to the weight values of each vertex point. After the BBW deformation was done, the non-rigid ICP registration was applied to register the surface of the BBW-deformed template to the surface of the target image. Figure 2 illustrates the non-rigid ICP registration of the template model to different target images.

The application of the template registration resulted in the same number of vertex points and a consistent structure of mesh [12-14] across all the 3D images in the database. As the hybrid registration approach that considers the landmark locations derives the same mesh topology across all the template-registered faces or heads, our 


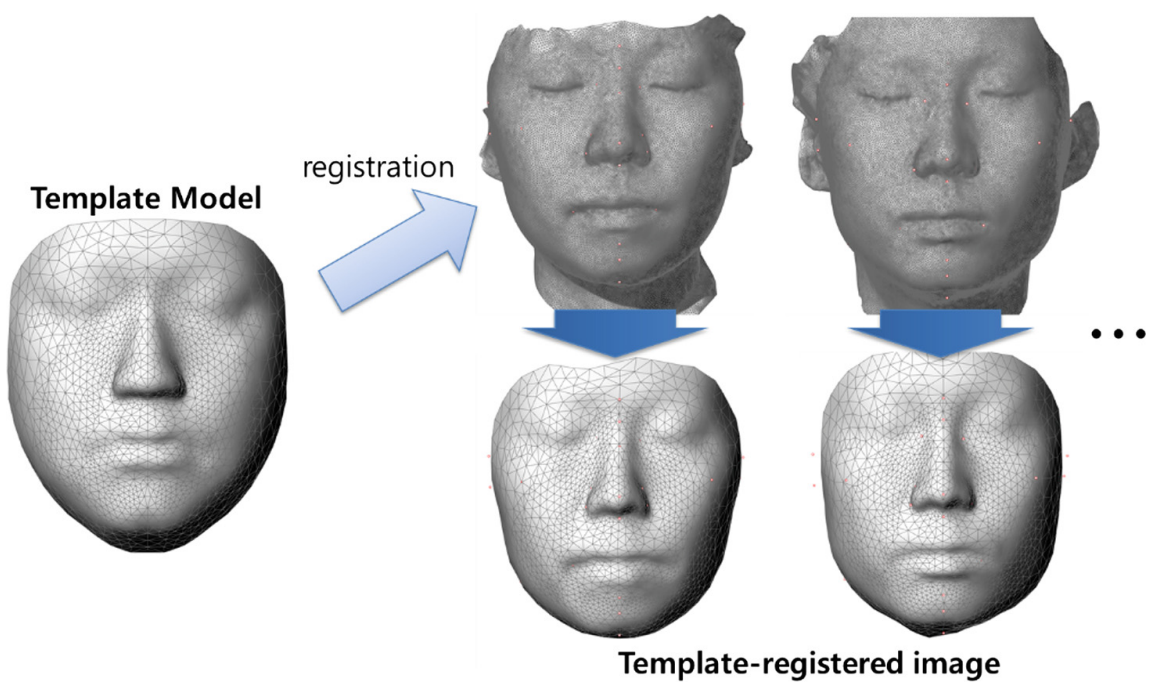

Fig. 2. Template registration of the face (illustrated).
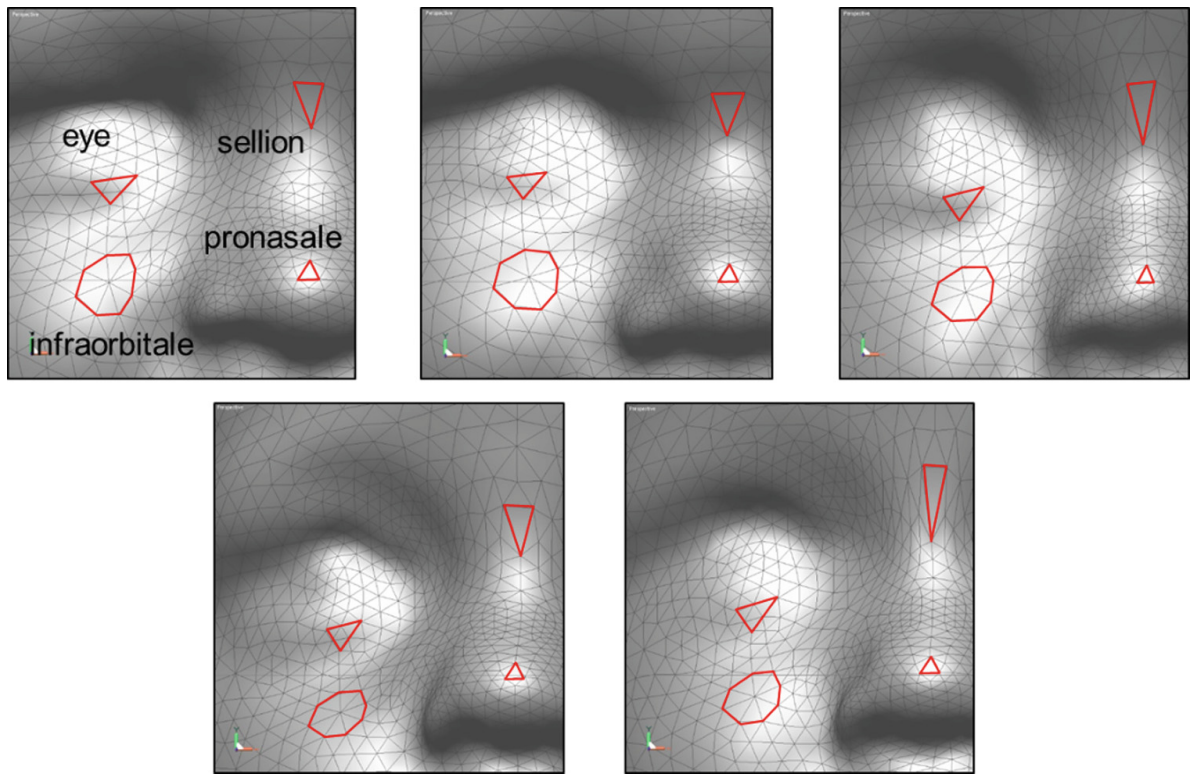

Fig. 3. Consistency of mesh structure among template-registered images.

method could provide consistency between template-registered images (Fig. 3). The BBW deformation and non-rigid ICP registration were conducted using the Matlab (MathWorks, Inc., Natick, MA, USA) programming language. 


\subsection{Facial Wearable Products}

By 3D scanning of a product, a curvy shape that is contacting to a facial area was saved as a spline curvature. A facial wearable product was 3D scanned using Artec Eva 3D scanner (Artec Group, Inc., Luxembourg). Then, based on the scanned image, the curve of the edge contacting to the face was drawn as the spline curvature as shown in Fig. 4. The pilot oxygen mask and three different VR headsets were used in this study.

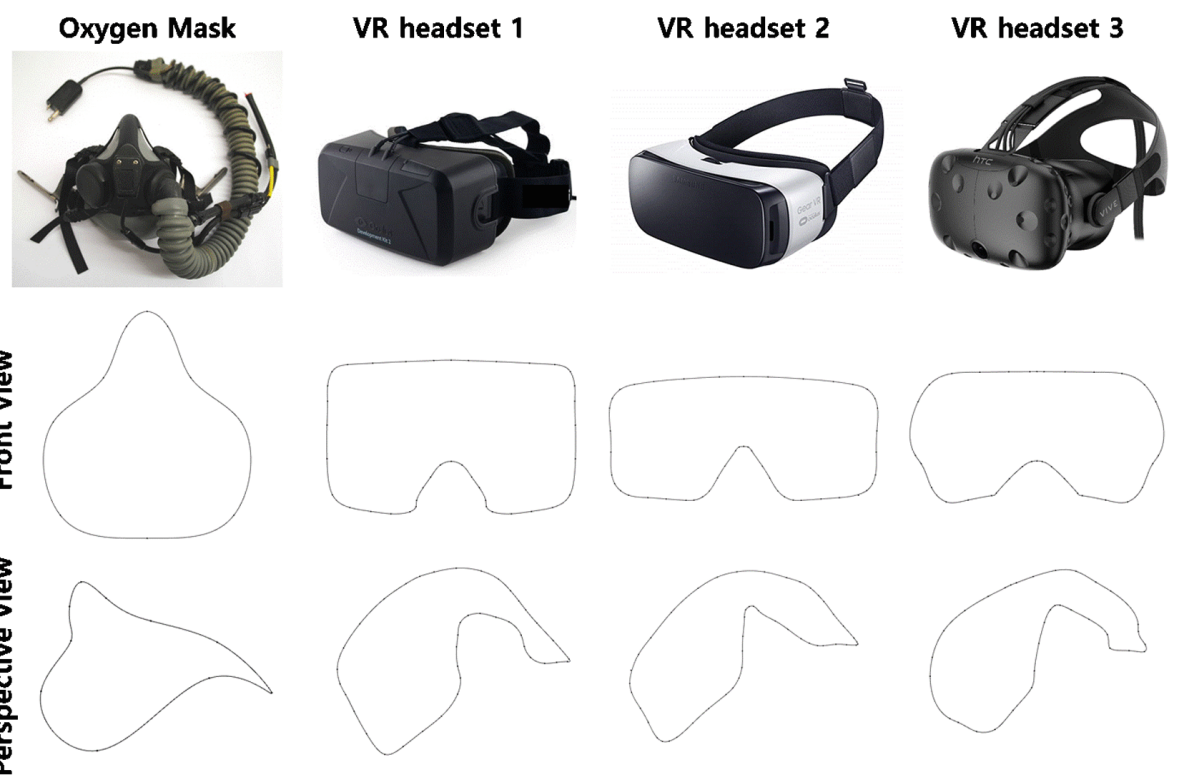

Fig. 4. Facial wearable products and its spline curvature in front and perspective views.

\subsection{Contact Pressure Estimation}

When the prepared spline curvature is positioned on a template-registered face, the contact pressure of the curvature towards a 3D face was calculated using finite element analysis [15]. In this work, we applied triangular shell elements for the 3D facial modeling based on the 3D scanned image data as shown in Fig. 1. The face model consists of 2,624 elements (the element in FE is the same concept to the mesh) and 1,340 nodes (the node in FE is the same concept to the vertex) and the head model consists of 5,532 elements and 2,787 nodes. Since the contact pressure estimation of the facial skin with small deformation was mainly focused on, the simple linear elastic material properties were applied on the nodes except some fixed nodes which are unmovable. For static analysis, the following equation was used.

$$
f=K u(N)
$$


where, $K$ is a global stiffness matrix, $u$ is a displacement vector for all nodes, and $f$ is a force vector (unit: $N$ ) for all nodes, respectively. The Young's elastic modulus $(E)$, Poisson ratio $(v)$, and the thickness of the triangular shell, which are necessary for the calculation of the $K$ are defined as $0.03,0.5$, and $2 \mathrm{~mm}$, representatively, by referring to previous studies [16-21].

The displacement vector $u$ was derived as the Euclidean distance between the curvature and each node. In this study, only $z$-translation out of $u$ vectors at each nodal coordinate was simply considered with the stiffness matrix $K$ to compute forces, which horizontally affects toward the face. By solving Eq. (1), the contact pressure $f$ was calculated. The contact pressure is calculated as the force exerted on a surface divided by the area $\left(\mathrm{mm}^{2}\right)$ over where the force is applied (Eq. 2). Figure 5 illustrates the estimated contact pressure with intensity level by the red color. The pressure calculation process was implemented in the Matlab environment.

$$
P=f / A\left(N / \mathrm{mm}^{2}=\mathrm{MPa}\right)
$$

where, $P$ is a contact pressure vector for all nodes, $f$ is a force vector (unit: $N$ ) for all nodes, and $A$ is an area vector (unit: $\mathrm{mm}^{2}$ ) for all nodes, respectively. The contact pressure value at a single node could be derived by dividing the applied force at the node by the sum of areas of meshes that include the node (Eq. 3).

$$
A_{i}=\frac{1}{3} \sum_{j=1}^{n} \frac{\left|\overrightarrow{N_{j 1} N_{j 2}} \times \overrightarrow{N_{j 1} N_{j 3}}\right|}{2}, \quad j=\{1,2,3, \ldots, n\}\left(\mathrm{mm}^{2}\right)
$$

where, $A_{i}$ is an area for a node $i, N_{j 1}$ is the node $i$ of a mesh $j, N_{j 2}$ and $N_{j 3}$ is the remaining two other nodes of a mesh $j$, and $n$ is the total number of meshes which include a node $i$. The sum of areas of each mesh is divided by 3 due to only one node (node $i$ ) out of three nodes in a mesh is used to calculate the contact pressure for the node $i\left(P_{i}\right)$.

\section{Results}

The average, min, max, and variation of the predicted facial contact pressure of the pilot oxygen mask for all the template-registered faces were analyzed. The mean contact pressure was 0.001 to $0.092 \mathrm{MPa}$ (average $\pm \mathrm{SD}=0.010 \pm 0.014 \mathrm{MPa}$ ) by face area for an oxygen mask design (Fig. 6). While the highest contact pressure was applied at nasal side area (maximum 0.09 $\mathrm{MPa}$ ), the lowest contact pressure was shown at chin area (around 0.01 MPa on average). For the VR headsets, the contact pressure showed differently by design of headsets under similar fitting condition (slightly fit each headset's spline curvature at the forehead and nose, then push it $10 \mathrm{~mm}$ toward the face). The contact pressure around the nose area shows higher than other contact areas, but the level of pressure is different. The VR headset 1, 2, and 3 showed a maximum of $0.05,0.07$, and $0.11 \mathrm{MPa}$ at nose area, respectively (Fig. 7). 


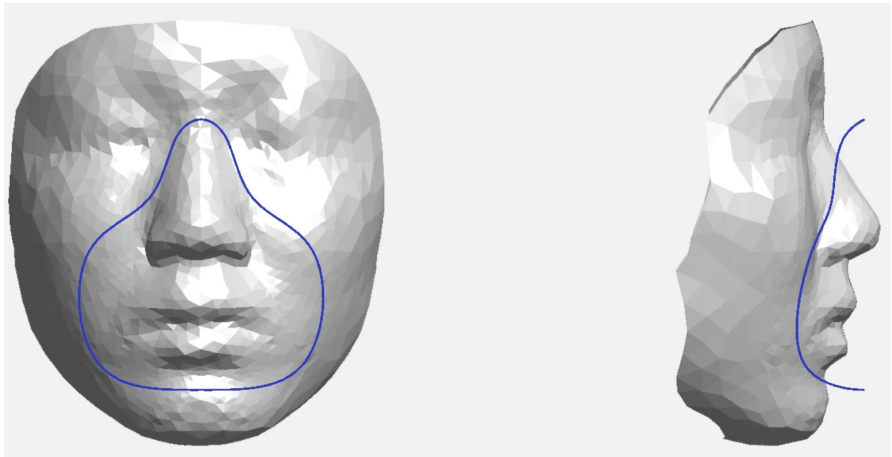

(a) Face without contacting to the mask curvature: no pressure.

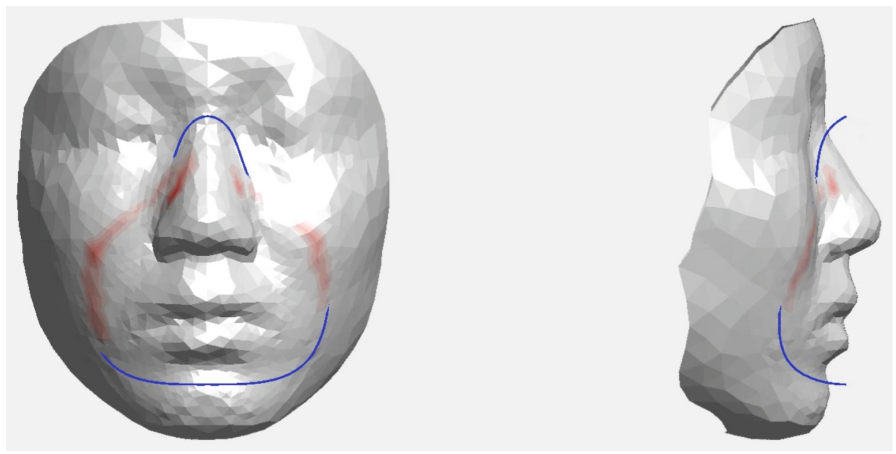

(b) Face with partially contacting mask

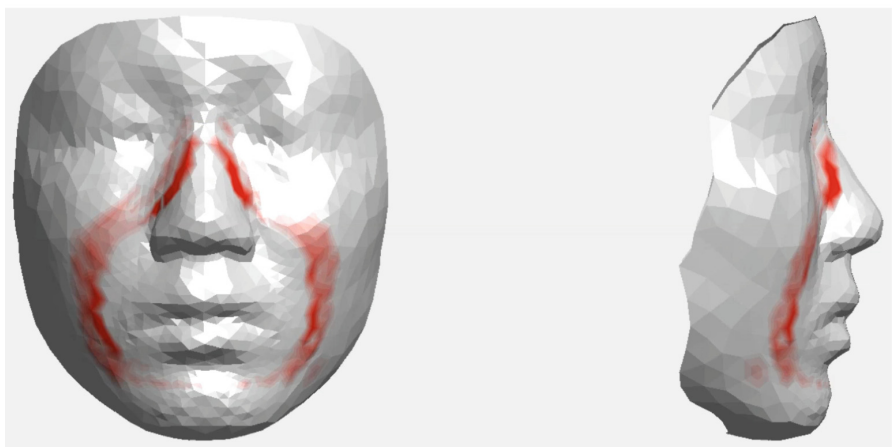

(c) Face with fully contacting mask

Fig. 5. The estimated contact pressure between the template-registered face and the mask defined as a curvy shape (illustrated). 


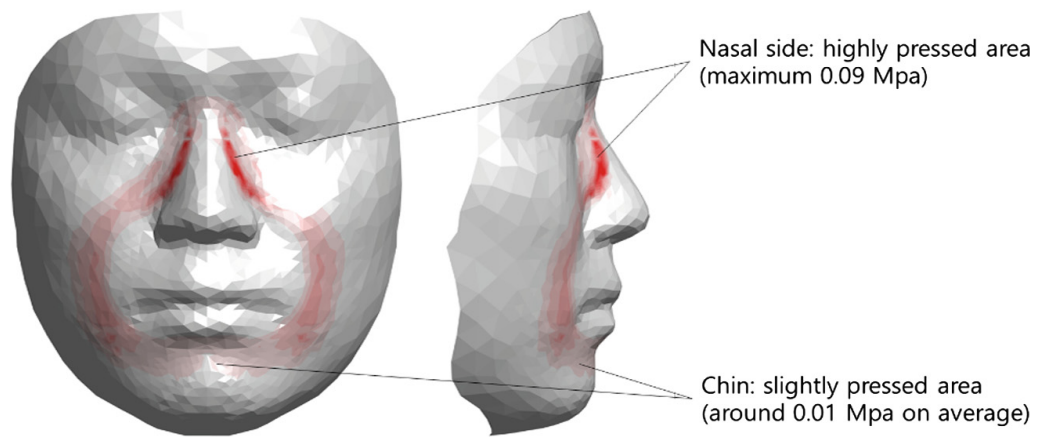

Fig. 6. Average contact pressure of oxygen mask (illustrated).

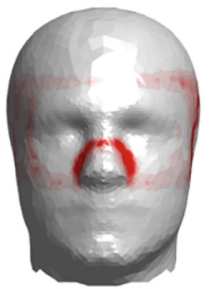

(a) VR headset 1
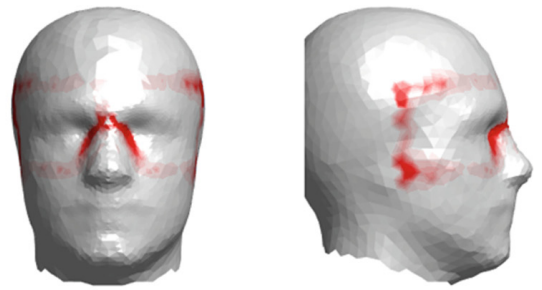

(b) VR headset 2
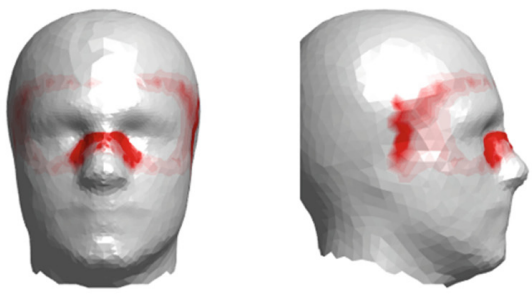

(c) VR headset 3

Fig. 7. Average contact pressure of virtual reality headsets (illustrated).

\section{Discussion}

This study introduces a novel product design approach using template model registration and FE analysis methods based on 3D body scan database. As our previous research, the virtual fit analysis method $[1,2,22]$ was proposed, which assessed the fit of an oxygen mask design (which was defined as spline curvature, either) onto 3D human faces by calculating Euclidean distances between the mask and facial surface. In the virtual fit analysis method, any material properties of both the face and mask were not considered; however, the prototype of the new oxygen mask designed based on the virtual fit analysis showed good fit and comfort compared to the existing one [23]. That was because not only a few facial dimensions but also the numerous facial shapes could 
be considered when the new design was found. In addition, by applying the template registration and $\mathrm{FE}$ analysis techniques with the virtual fitting method, the contact pressure characteristics could be considered in product design.

Compare to the previous FE-based studies, this study used simplified FE approach for quick analysis of the contact pressure between hundreds or thousands of 3D human scan images and several different product designs. Making a more realistic FE model made using an FE analysis software is time-consuming and not easy to be applied to hundreds of 3D scan images due to a complex manual procedure. So that only a few body images were used in previous studies. Instead of using an FE software, this study used Matlab-programmed a simple FE model that is using the triangular shell. Consequently, the FE model using the triangular shell can be directly applied to mesh structures of the 3D scan data. Therefore, our FE approach does not require any manual works, but the computer automatically calculates the FE process and the analysis of contact pressure. By this approach, the complex FE analysis method could be conducted in reasonably short computation time (say, less than a minute per $3 \mathrm{D}$ scan) to calculate all the numerous $3 \mathrm{D}$ scan data.

The template registration method was used in order to make all 3D scan images having the same number of vertex points and the same mesh structure. A hybrid template registration method consisting of the BBW and non-rigid ICP algorithms was applied in this study. In the proposed hybrid approach, the template model is first roughly matched to a target model based on the location of landmarks using the BBW algorithm; then, the registration is conducted. Through the hybrid approach, the structure of meshes surrounding each landmark shows high consistency across all template-registered face/head models.

There are some limitations in the proposed FE approach, which need to be further studied to make the model more accurate and valid in terms of the prediction of the contact pressure. While the conventional FE analysis uses a force vector $(f)$ to calculate a displacement vector $(u)$ and other relevant outcomes such as stress and strain; contrariwise, this study used the displacement vector to calculate the force vector and contact pressure. The displacement in this study means a pushed-in distance of the skin surface caused by the positioning of the spline curvature toward the face. But, the position of the spline curvature was somewhat artificial and might not be very realistic, although the resulted contact pressure values were quite reasonable compare to the previous study $[23,24]$. The more realistic way of fitting the spline curvature to the faces needs to be studied. A method of analysis of the contact pressure by different facial areas also needs to be studied. In addition, differentiation of Young's modulus and Poisson ratio values for different facial areas are also considered. An empirical approach might be required to validate the FE model. Nevertheless, the current FEbased method needs to be further improved to be more realistic and robust, the proposed approach could be usefully applied for analysis and design of wearable products that fit the human body.

Acknowledgments. This work was jointly supported by the National Program for Excellence in Software and by the National Research Foundation of Korea (NRF) grants funded by the Ministry of Science and ICT (2017-0-00130; 2018R1C1B5047805). 


\section{References}

1. Lee, W., Kim, H., Jung, D., Park, S., You, H.: Ergonomic design and evaluation of a pilot oxygen mask. In: Human Factors and Ergonomics Society 57th Annual Meeting (2013)

2. Lee, W., Yang, X., Jung, H., You, H., Goto, L., Molenbroek, J.F.M., Goossens, R.H.M.: Application of massive 3D head and facial scan datasets in ergonomic head-product design. Int. J. Digit. Hum. 1(4), 344-360 (2016)

3. Lee, W., Kim, J.-G., Molenbroek, J.M.F., Goossens, R.H.M., Jung, H., You, H.: Contact pressure analysis for wearable product design. In: Proceedings of the 20th Congress of the International Ergonomics Association (IEA 2018), pp. 163-169 (2019)

4. Dai, J.C., Yang, J.Z., Zhuang, Z.Q.: Sensitivity analysis of important parameters affecting contact pressure between a respirator and a headform. Int. J. Ind. Ergon. 41(3), 268-279 (2011)

5. Lei, Z.P., Yang, J., Zhuang, Z.Q., Roberge, R.: Simulation and evaluation of respirator faceseal leaks using computational fluid dynamics and infrared imaging. Ann. Occup. Hyg. 57(4), 493-506 (2013)

6. Lei, Z.P., Yang, J., Zhuang, Z.Q.: A novel algorithm for determining contact area between a respirator and a headform. J. Occup. Environ. Hyg. 11(4), 227-237 (2014)

7. Lee, W., Jeong, J., Park, J., Jeon, E., Kim, H., Jung, D., Park, S., You, H.: Analysis of the facial measurements of Korean Air Force pilots for oxygen mask design. Ergonomics 56(9), 1451-1464 (2013)

8. Lee, W., Lee, B., Yang, X., Jung, H., Bok, I., Kim, C., Kwon, O., You, H.: A 3D anthropometric sizing analysis system based on North American CAESAR 3D scan data for design of head wearable products. Comput. Ind. Eng. 117, 121-130 (2018)

9. Lee, W., Goto, L., Molenbroek, J.F.M., Goossens, R.H.M.: Analysis methods of variations of facial size and shape using a 3D scanning for facial mask design. In: Human Factors and Ergonomics Society 61st Annual Meeting (2017)

10. Jacobson, A., Baran, I., Popović, J., Sorkine-Hornung, O.: Bounded biharmonic weights for real-time deformation. Commun. ACM 57(4), 99-106 (2014)

11. Besl, P.J., McKay, N.D.: A method for registration of 3-D shapes. IEEE Trans. Pattern Anal. Mach. Intell. 14(2), 239-256 (1992)

12. Allen, B., Curless, B., Popović, Z.: The space of human body shapes: reconstruction and parameterization from range scans. ACM Trans. Graph. 22(3), 587-594 (2003)

13. Tsoli, A., Loper, M., Black, M.J.: Model-based anthropometry: predicting measurements from 3D human scans in multiple poses. In: IEEE Winter Conference on Applications of Computer Vision (WACV) (2014)

14. Xi, P., Shu, C.: Consistent parameterization and statistical analysis of human head scans. Vis. Comput. 25(9), 863-871 (2009)

15. Bathe, K.-J.: Finite Element Procedures, 3rd edn. K.J. Bathe, Watertown (2007)

16. McKee, C.T., Last, J.A., Russell, P., Murphy, C.J.: Indentation versus tensile measurements of Young's modulus for soft biological tissues. Tissue Eng. Part B Rev. 17(3), 155-164 (2011)

17. Xu, M., Yang, J.: Human facial soft tissue thickness and mechanical properties: a literature review. In: Volume 1A: 35th Computers and Information in Engineering Conference (2015)

18. Luboz, V., Promayon, E., Payan, Y.: Linear elastic properties of the facial soft tissues using an aspiration device: towards patient specific characterization. Ann. Biomed. Eng. 42(11), 2369-2378 (2014)

19. Kalra, A., Lowe, A., Al-Jumaily, A.M.: Mechanical behaviour of skin: a review. J. Mater. Sci. Eng. 5(4), (2016) 
20. Choi, A.P.C., Zheng, Y.P.: Estimation of Young's modulus and Poisson's ratio of soft tissue from indentation using two different-sized indentors: finite element analysis of the finite deformation effect. Med. Biol. Eng. Comput. 43(2), 258-264 (2005)

21. Chopra, K., Calva, D., Sosin, M., Tadisina, K.K., Banda, A., De La Cruz, C., Chaudhry, M.R., Legesse, T., Drachenberg, C.B., Manson, P.N., Christy, M.R.: A comprehensive examination of topographic thickness of skin in the human face. Aesthetic Surg. J. 35(8), 1007-1013 (2015)

22. Lee, W., Lee, B., Kim, S., Jung, H., Jeon, E., Choi, T., You, H.: 3D scan to product design: methods, techniques, and cases. In: 6th International Conference on 3D Body Scanning Technologies (2015)

23. Lee, W., Yang, X., Jung, D., Park, S., Kim, H., You, H.: Ergonomic evaluation of pilot oxygen mask designs. Appl. Ergon. 67, 133-141 (2018)

24. Lei, Z., Yang, J., Zhuang, Z.: Headform and N95 filtering facepiece respirator interaction: contact pressure simulation and validation. J. Occup. Environ. Hyg. 9(1), 46-58 (2012) 